\title{
The Relational Blockworld Interpretation of Non-relativistic Quantum Mechanics
}

\author{
W.M. Stuckey ${ }^{1}$, Michael Silberstein ${ }^{2,3}$ and Michael Cifone ${ }^{3}$ \\ 1. Department of Physics, Elizabethtown College, Elizabethtown, PA, USA 17022 \\ stuckeym@etown.edu \\ 2. Department of Philosophy, Elizabethtown College, Elizabethtown, PA, USA 17022 \\ silbermd@etown.edu \\ 3. Department of Philosophy, University of Maryland, College Park, MD, USA 20742 \\ cifonemc@wam.umd.edu
}

\begin{abstract}
We introduce a new interpretation of non-relativistic quantum mechanics (QM) called Relational Blockworld (RBW). We motivate the interpretation by outlining two results due to Kaiser, Bohr, Ulfeck, Mottelson, and Anandan, independently. First, the canonical commutation relations for position and momentum can be obtained from boost and translation operators, respectively, in a spacetime where the relativity of simultaneity holds. Second, the QM density operator can be obtained from the spacetime symmetry group of the experimental configuration exclusively. We show how QM, obtained from relativistic quantum field theory per RBW, explains the twin-slit experiment and conclude by resolving the standard conceptual problems of QM, i.e., the measurement problem, entanglement and non-locality.
\end{abstract}

Keywords: Blockworld, non-relativistic quantum mechanics, measurement problem, entanglement.

PACS: 03.65.Ta; 03.65.Ud.

\section{INTRODUCTION}

Referring to quantum mechanics, Murray Gell-Mann writes [1], "We all know how to use it and how to apply it to problems; and so we have learned to live with the fact that nobody can understand it." In seeking to "understand it," we introduce a purely geometric (acausal and adynamical) account of non-relativistic quantum mechanics (QM) called Relational Blockworld. The ontology of this interpretation is one in which constructive objects (entities such as particles or waves with worldlines in spacetime) are not fundamental constituents of reality. Rather, constructive objects are composed of spatiotemporal relations and physical phenomena are described by the distribution of spatiotemporal relations throughout space and time as a whole, i.e., in the blockworld. This represents a radical departure from physics per the dynamical perspective, e.g., particles of force exchanged by particles of matter to explain phenomena.

We justify this new worldview by outlining a result obtained independently by Kaiser [2], Bohr \& Ulfbeck [3] and Anandan [4] which shows the Heisenberg commutation relations of QM follow from the relativity of simultaneity in the $c \rightarrow \infty$ limit of the Poincaré group. We believe this legitimizes the use of a blockworld for the 
ontology of non-relativisitic quantum mechanics. That relations, rather than objects/substances with worldlines, are fundamental in the blockworld is justified by the work of Bohr, Mottleson \& Ulfbeck [5] who showed that the QM density matrix can be obtained from the spacetime symmetry group of the experimental configuration alone. We also outline their result herein.

In order to emphasize that no thing propagates through the space between source and detector to cause an experimental "click," we relate QM to relativistic quantum field theory (RQFT) per RBW and explain the twin-slit experiment. Per our ontology, the continuous distribution of oscillators in space (the field) per RQFT is replaced by a discrete, rarified distribution of RQFT "sources" alone (one at the experimental source, one at each slit, and one at the detector in the twin-slit experiment). The transition amplitude relating these discretely distributed RQFT sources and oscillators then provides QM's familiar wave function giving rise to the twin-slit interference pattern per the Born rule.

We conclude by using RBW to resolve the standard conceptual problems of QM, i.e., entanglement, non-locality and the measurement problem. An important consequence of this resolution is that we do not have to compromise relativity theory. Quite the contrary, per RBW the most vexing implication of special relativity - the lack of a moving Now due to the relativity of simultaneity (to which Einstein was "painfully resigned" [6]) - is also responsible for QM's “only mystery" (per Feynman [7]), i.e., quantum interference. The trick is to appreciate that everything resides in a 4D spacetime and nowhere else, and quantum phenomena cannot be modeled with worldlines if one is to do justice to its non-commutative structure. Thus while clicks in detectors are perfectly classical events, the clicks are not evidence of constructive quantum entities such as particles or waves with worldlines. Rather, the clicks are manifestations of spacetime relations distributed among the elements of the experimental configuration per the spacetime symmetries. Such acausal, global determination relations do not respect any common cause principle. This fact should not bother anyone who has truly transcended the idea that the dynamical or causal perspective is the most fundamental one.

\section{COMMUTATION RELATIONS AND RELATIVITY OF SIMULTANEITY}

Kaiser has shown that the non-commutivity of Lorentz boosts with spatial translations is responsible for the non-commutivity of the quantum mechanical position operator with the quantum mechanical momentum operator. He writes ${ }^{1}$,

For had we begun with Newtonian spacetime, we would have the Galilean group instead of [the restricted Poincaré group]. Since Galilean boosts commute with spatial translations (time being absolute), the brackets between the corresponding generators vanish, hence no canonical commutation relations (CCR)! In the [c $\rightarrow \infty$ limit of the Poincare algebra], the CCR are a remnant of relativistic invariance

\footnotetext{
${ }^{1}$ Kaiser (1981), p. 706.
} 
where, due to the nonabsolute nature of simultaneity, spatial translations do not commute with pure Lorentz transformations. [Italics his].

Bohr \& Ulfbeck ${ }^{2}$ also realized that the "Galilean transformation in the weakly relativistic regime" is needed to construct a position operator for QM, and this transformation "includes the departure from simultaneity, which is part of relativistic invariance." Specifically, they note that the commutator between a "weakly relativistic" boost and a spatial translation results in "a time displacement," which is crucial to the relativity of simultaneity. Thus they write ${ }^{3}$,

"For ourselves, an important point that had for long been an obstacle, was the realization that the position of a particle, which is a basic element of non-relativistic quantum mechanics, requires the link between space and time of relativistic invariance."

So, the essence of non-relativistic quantum mechanics - its canonical commutation relations $-i$ s entailed by the relativity of simultaneity.

To outline Kaiser's result, we take the limit $c \rightarrow \infty$ in the Lie algebra of the Poincaré group for which the non-zero brackets are

$$
\begin{gathered}
{\left[J_{m}, J_{n}\right]=i J_{k}} \\
{\left[T_{0}, K_{n}\right]=i T_{n}} \\
{\left[K_{m}, K_{n}\right]=\frac{-i}{c^{2}} J_{k}} \\
{\left[J_{m}, K_{n}\right]=i K_{k}} \\
{\left[J_{m}, T_{n}\right]=i T_{k}} \\
{\left[T_{m}, K_{n}\right]=\frac{-i}{c^{2}} \delta_{m n} T_{0}}
\end{gathered}
$$

where expressions with subscripts $\mathrm{m}, \mathrm{n}$ and $\mathrm{k}$ denote 1,2 and 3 cyclic, $J_{\mathrm{m}}$ are the generators of spatial rotations, $T_{0}$ is the generator of time translations, $T_{\mathrm{m}}$ are the generators of spatial translations, $K_{\mathrm{m}}$ are the boost generators, $i^{2}=-1$, and $c$ is the speed of light. We obtain

$$
\begin{gathered}
{\left[J_{m}, J_{n}\right]=i J_{k}} \\
{\left[M, K_{n}\right]=0} \\
{\left[K_{m}, K_{n}\right]=0} \\
{\left[J_{m}, K_{n}\right]=i K_{k}} \\
{\left[J_{m}, T_{n}\right]=i T_{k}} \\
{\left[T_{m}, K_{n}\right]=\frac{-i}{\hbar} \delta_{m n} M}
\end{gathered}
$$

${ }^{2}$ Bohr \& Ulfbeck (1995), section D of part IV, p. 28.

${ }^{3}$ Ibid., p. 24. 
where $M$ is obtained from the mass-squared operator in the $c \rightarrow \infty$ limit since

$$
c^{-2} \hbar T_{0}=c^{-2} P_{0}
$$

and

$$
\frac{P_{o}}{c^{2}}=\left(M^{2}+c^{-2} P^{2}\right)^{1 / 2}=M+\frac{P^{2}}{2 M c^{2}}+O\left(c^{-4}\right) .
$$

Thus, $c^{-2} T_{0} \rightarrow \frac{M}{\hbar}$ in the limit $c \rightarrow \infty$. [M $\equiv m I$, where $m$ is identified as "mass" by choice of 'scaling factor' $\hbar$.] So, letting

and

$$
P_{s} \equiv \hbar T_{s}
$$

$$
Q_{n} \equiv \frac{-\hbar}{m} K_{n}
$$

we have

$$
\left[P_{s}, Q_{n}\right]=\frac{-\hbar^{2}}{m}\left[T_{s}, K_{n}\right]=\left(\frac{-\hbar^{2}}{m}\right)\left(\frac{i}{\hbar}\right) \delta_{s n} m I=-i \hbar \delta_{s n} I
$$

Bohr \& Ulfbeck point out that in this "weakly relativistic regime" the coordinate transformations now look like

$$
\begin{aligned}
& X=x-v t \\
& T=t-\frac{v x}{c^{2}}
\end{aligned}
$$

These transformations differ from Lorentz transformations because they lack the factor

$$
\gamma=\left(1-\frac{v^{2}}{c^{2}}\right)^{-1 / 2}
$$

which is responsible for time dilation and length contraction. And, these transformations differ from Galilean transformations by the temporal displacement $v x / c^{2}$ which is responsible for the relativity of simultaneity, i.e., in a Galilean transformation time is absolute so $T=t$. Therefore, the spacetime structure of Kaiser et al. lies between Galilean spacetime and Minkowski spacetime and we see that the Heisenberg commutation relations are not the result of Galilean invariance, where spatial translations commute with boosts, but rather they result from the relativity of simultaneity per Lorentz invariance.

Why is it that the dynamics of QM, given by the Schrödinger equation, are Galilean invariant? That is, why are the dynamics of QM unaffected by the relativity of simultaneity reflected in the geometry of Eq. (1)? To answer this question we operate on $|\psi\rangle$ first with the spatial translation operator then the boost operator and compare that outcome to the reverse order of operations. The spatial translation (by $a$ ) and boost (by $v$ ) operators in $x$ are 


$$
U_{T}=\exp \left(-i a T_{x}\right) \text { and } U_{K}=\exp \left(-i v K_{x}\right)
$$

respectively. These yield

$$
U_{K} U_{T}|\psi\rangle=U_{T} U_{K} \exp (\operatorname{iavmI} / \hbar)|\psi\rangle
$$

Thus, we see that the geometric structure of Eq. (1) introduces a mere phase to $|\psi\rangle$ and is therefore without consequence in the computation of expectation values. And in fact, this phase is consistent with that under which the Schrödinger equation is shown to be Galilean invariant $[8,9]$.

\section{DENSITY MATRIX FROM SYMMETRY GROUP}

To show how, in general, one may obtain the density matrix using only the irreducible representations (irreps) of the symmetry group $D(g)$ and their averages $<D(g)>$, we start with Eq. (1.68) of Georgi [10]

$$
\sum_{g} \frac{n_{a}}{N}\left[D_{a}\left(g^{-1}\right)\right]_{k j}\left[D_{b}(g)\right]_{l m}=\delta_{a b} \delta_{j l} \delta_{k m}
$$

where $n_{a}$ is the dimensionality of the irrep, $D_{a}$, and $N$ is the group order. If we consider but one particular irrep, $D$, this reduces to the orthogonality relation of Bohr et al.

$$
\sum_{g} \frac{n}{N}\left[D\left(g^{-1}\right)\right]_{k j}[D(g)]_{l m}=\delta_{j l} \delta_{k m}
$$

where $n$ is the dimension of the irrep. Now multiply by $[D(g)]_{j k}$ and sum over $\mathrm{k}$ and $\mathrm{j}$ to obtain

$$
\sum_{j} \sum_{k} \sum_{g} \frac{n}{N}\left[D\left(g^{-1}\right)\right]_{k j}[D(g)]_{l m}\left[D\left(g^{\prime}\right)\right]_{j k}=\sum_{j} \sum_{k} \delta_{j l} \delta_{k m}\left[D\left(g^{\prime}\right)\right]_{j k}=\left[D\left(g^{\prime}\right)\right]_{l m}
$$

The first sum on the LHS gives

$$
\sum_{j}\left[D\left(g^{-1}\right)\right]_{k j}\left[D\left(g^{\prime}\right)\right]_{j k}=\left[D\left(g^{-1}\right) D\left(g^{\prime}\right)\right]_{k k}
$$

The sum over $\mathrm{k}$ then gives the trace of $D\left(g^{-1}\right) D(g)$, so we have

$$
\frac{n}{N} \sum_{g}[D(g)]_{l m} \operatorname{Tr}\left\{D\left(g^{-1}\right) D\left(g^{\prime}\right)\right\}=\left[D\left(g^{\prime}\right)\right]_{l m}
$$

which Bohr et al. write without subscripts, i.e.,

$$
\frac{n}{N} \sum_{g} D(g) \operatorname{Tr}\left\{D\left(g^{-1}\right) D\left(g^{\prime}\right)\right\}=D\left(g^{\prime}\right) .
$$

If in a particular experiment we measure directly the click distributions associated with the various eigenvalues of a symmetry $D(g)$, we obtain its average outcome 


$$
\langle D(g)\rangle=\sum_{i} \lambda_{i} p\left(\lambda_{i}\right)
$$

where $\lambda_{i}$ are the eigenvalues of $D(g)$ and $p\left(\lambda_{i}\right)$ are the distribution frequencies for the observations of the various eigenvalues/outcomes.

In terms of averages, Eq. (6) becomes

$$
\frac{n}{N} \sum_{g}\langle D(g)\rangle \operatorname{Tr}\left\{D\left(g^{-1}\right) D\left(g^{\prime}\right)\right\}=\left\langle D\left(g^{\prime}\right)\right\rangle
$$

Since we want the density matrix to satisfy the standard relation

$$
\operatorname{Tr}\left\{\rho D\left(g^{\prime}\right)\right\}=\left\langle D\left(g^{\prime}\right)\right\rangle
$$

it must be the case that

$$
\rho \equiv \frac{n}{N} \sum_{g} D\left(g^{-1}\right)\langle D(g)\rangle
$$

That this density operator is hermitian follows from the fact that the symmetry operators are unitary. That is, $D\left(g^{-1}\right)=D^{\dagger}(g)$ implies $\left\langle D\left(g^{-1}\right)\right\rangle=\langle D(g)\rangle^{*}$, thus

$$
\rho^{+}=\frac{n}{N} \sum_{g} D^{+}\left(g^{-1}\right)\langle D(g)\rangle^{*}=\frac{n}{N} \sum_{g} D(g)\left\langle D\left(g^{-1}\right)\right\rangle=\frac{n}{N} \sum_{g} D\left(g^{-1}\right)\langle D(g)\rangle=\rho .
$$

[The second-to-last equality holds because we are summing over all $g$ and for each $g$ there exists $g^{-1}$.] So, the density operator of Eq. (10) will be hermitian and, therefore, its eigenvalues (probabilities) are guaranteed to be real. This is not necessarily the case for $D(g)$, since we know only that they are unitary. However, we need only associate detector clicks with the eigenvalues of $D(g)$ and in this perspective one does not attribute an eigenvalue of $D(g)$ to a property of some 'click-causing particle'. Therefore, whether or not the eigenvalues of any particular $D(g)$ are real or imaginary is of no ontological or empirical concern.

\section{TWIN-SLIT EXPERIMENT}

Given our geometrical interpretation of QM, it should be clear that we do not take detector events to be indicators of the trajectories of classical-like particles or wavefunctions, propagating from the source to the detector as in Bohm's mechanics or even, as it turns out, like disturbances in a field per RQFT. In RQFT for a scalar field without scattering we have for the transition amplitude [11]

$$
Z=\int D \varphi \exp \left[i \int d^{4} x\left[\frac{1}{2}(d \varphi)^{2}-V(\varphi)\right]\right]
$$


According to [11], QM then obtains in $(0+1)$ dimensions. In the derivation of Eq. (11) from $\mathrm{QM}$, the field $\varphi$ is obtained in the continuum limit of a discrete set of oscillators $q_{\mathrm{a}}$ distributed in a spatial lattice. Any one of these $q_{\mathrm{a}}$ is supposed to replace $\varphi$ in Eq. (11) in order that it reduce to QM. However, each $q_{\mathrm{a}}$ is fixed in space so the notion that we're integrating over all possible paths in space (standard treatment) from a source to a detector when we compute $\mathrm{Z}$ is not ontologically consistent with the fact that we integrate over all values of $q$ but not over all values of the index ' $\mathrm{a}$ ' in $q_{\mathrm{a}}$. We rather suggest that the method for reducing RQFT to QM is to associate sources $J(x)$ with elements in the experimental set up while assuming the $q$ 's are distributed discretely therein. Thus, we want to obtain QM from the discrete version of

$$
Z=\int D \varphi \exp \left[i \int d^{4} x\left[\frac{1}{2}(d \varphi)^{2}-V(\varphi)+J(x) \varphi(x)\right]\right]
$$

so we must compute

$$
Z=\int \ldots \int d q_{1} \ldots d q_{N} \exp \left[\frac{i}{2} q \cdot A \cdot q+i J \cdot q\right]
$$

where $A_{i j}$ is the discrete matrix counterpart to the dynamical differential operator and $J_{m}$ and $q_{n}$ are the discrete vector versions of $J(x, t)$ and $q(x, t)$, denoting their location in the spactime lattice. The solution to Eq. (13) for each path from $q_{\text {initial }}$ at the source to $q_{\text {final }}$ at some point on the detector is

$$
\left(\frac{(2 \pi i)^{N}}{\operatorname{det}(A)}\right)^{1 / 2} \exp \left[-\frac{i}{2} J \cdot A^{-1} \cdot J\right]
$$

For the twin-slit experiment, which "has in it the heart of quantum mechanics. In reality, it contains the only mystery" [7], we have four $J$ 's which must be taken into account when computing the amplitude between $q_{\text {initial }}$ and $q_{\text {final }}$, i.e., $J_{1} \rightarrow J_{2} \rightarrow J_{3}$ and $J_{1} \rightarrow J_{4} \rightarrow J_{3}$ (figure 1). The amplitude is then given by

$$
\psi \sim \exp \left[-\frac{i}{2}\left(J_{1} A_{12}^{-1} J_{2}+J_{2} A_{23}^{-1} J_{3}\right)\right]+\exp \left[-\frac{i}{2}\left(J_{1} A_{14}^{-1} J_{4}+J_{4} A_{43}^{-1} J_{3}\right)\right]
$$

Typically, the source is equidistance from either slit so $J_{1} A_{12}{ }^{-1} J_{2}=J_{1} A_{14}{ }^{-1} J_{4}$ and we have the familiar form

$$
\psi \sim \exp \left[-\frac{i}{2}\left(J_{2} A_{23}^{-1} J_{3}\right)\right]+\exp \left[-\frac{i}{2}\left(J_{4} A_{43}^{-1} J_{3}\right)\right]
$$




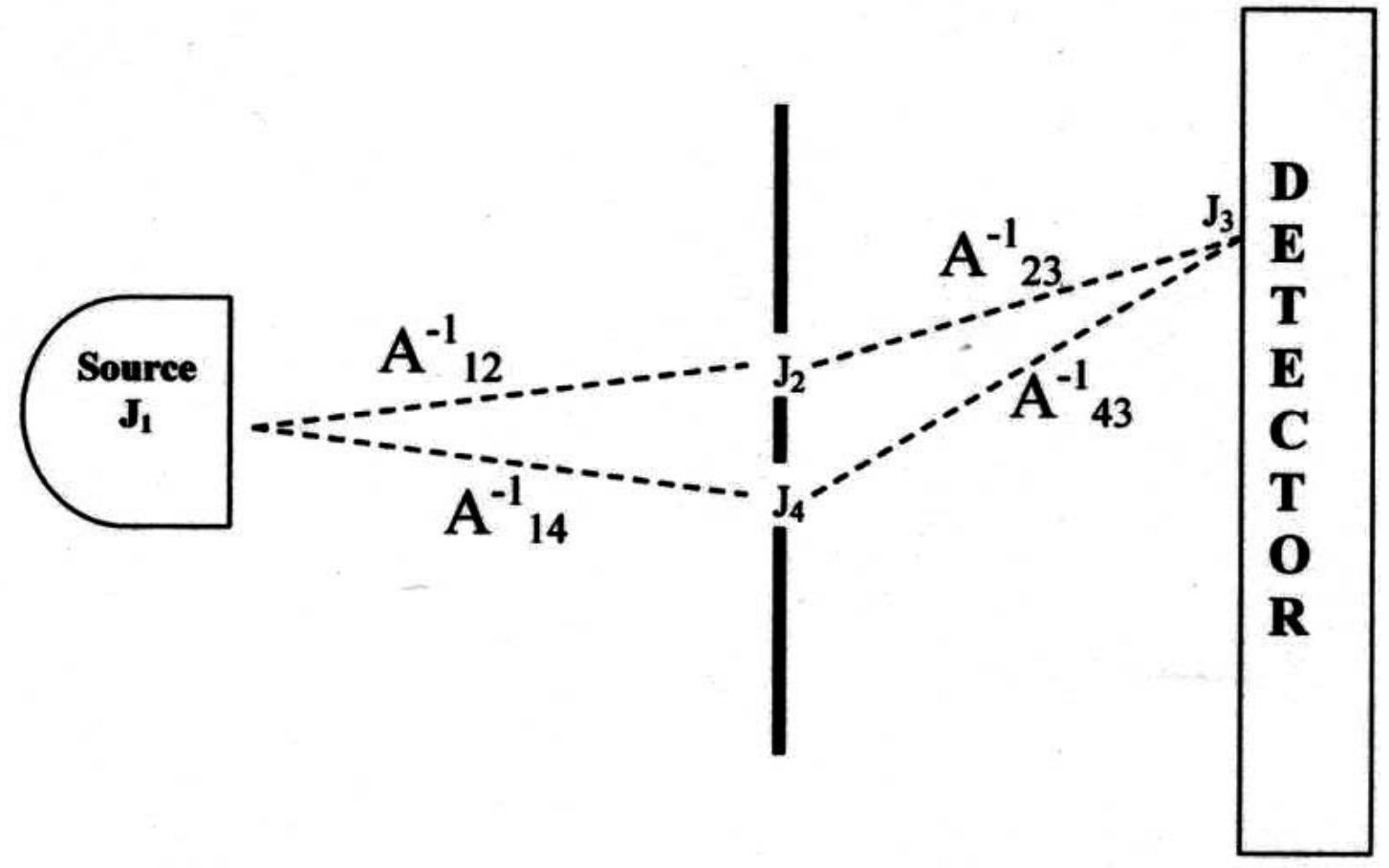

FIGURE 1. Twin-Slit Experiment

Since we are using the formalism of RQFT some might (erroneously) infer, in the parlance of particle physics, that we are creating a pair of particles at the source which propagate to each slit then annihilate at the detector. Recall however that we have a discrete distribution of $q$ 's at the source, slits and detector per QM rather than the continuous field $\varphi$ in the space between source, slits and detector. Thus it should be clear per our leitmotif that there is no particle (disturbance in the field per RQFT) propagating from the source through the slit(s) to the detector screen that causes a click thereupon. Rather the distribution of clicks at the detector evidences the spacetime relations (given here by $A_{i j}{ }^{-1}$ ) between the various elements of the experimental arrangement, i.e., the only 'things' with ontic status.

\section{RESOLVING THE CONCEPTUAL PROBLEMS OF QM}

\section{The Measurement Problem}

According to the account developed here, we offer a deflation of the measurement problem with a novel form of a hidden-variables "statistical interpretation." The fundamental difference between our version of this view and the usual understanding of it is the following: whereas on the usual view the state description refers to an "ensemble" which is an ideal collection of similarly prepared quantum particles, "ensemble" according to our view is just an ideal collection of spacetime regions $\mathrm{D}_{\mathrm{i}}$ 
"prepared" with the same spatiotemporal boundary conditions per the experimental configuration itself. The union of the click events in each $D_{i}$, as $i \rightarrow \infty$, produces the characteristic Born distribution. Accordingly, probability on our geometrical QM is interpreted per relative frequencies. It should be clear, also, that probabilities are understood as the likelihood that particular relations between the source and detector in spacetime are realized, from among a set of all equally likely relations between the source and detector.

On our view, the wave-function description of a quantum system can be interpreted statistically because we now understand that, as far as measurement outcomes are concerned, the Born distribution has a basis in the spacetime symmetries of experimental configuration. Each "click," which some would say corresponds to the impingement of a particle onto a measurement device with probability computed from the wave-function, corresponds to spacetime relations in the context of the experimental configuration. The measurement problem exploits the possibility of extending the wave-function description from the quantum system to the whole measurement apparatus, whereas the spacetime description according to RBW already includes the apparatus via the spacetime symmetries instantiated by the entire experimental configuration. The measurement problem is therefore a non-starter on our view.

\section{Entanglement \& Non-locality}

On our geometric view of QM each detection event, which evidences spacetime relations between source and detector, selects a trajectory from a family of possible trajectories (one family per entangled 'particle'). In the language of detection events qua relations, it follows that correlations are correlations between the members of the families of trajectories and these correlations are the result of the relevant spacetime symmetries for the experimental configuration. And, since an experiment's spacetime symmetries are manifested in the Hamilton-Jacobi families of trajectories throughout the relevant spacetime region $\mathrm{D}$, there is no reason to expect entanglement to diminish with distance from the source. Thus, the entanglement of families of trajectories is spatiotemporally global, i.e., non-local. That is, there is no reason to expect entanglement geometrically construed to respect any kind of common cause principle. Obviously, on our geometric interpretation there is no non-locality in the odious sense we find in Bohm for example, that is, there are no instantaneous causal connections (construed dynamically or in terms of production-bringing new states of affairs into being) between space-like separated events - no action at a distance. However our view is non-local in the sense that it violates the locality principle. The locality principle states: the result of a measurement is probabilistically independent of actions performed at space-like separation from the measurement. Keep in mind that in our blockworld setting, talk of "actions performed" gets only a purely logicalcounterfactual meaning - the entire experimental EPR set-up, its past, present and future, and the spacetime symmetries of that set-up are all just 'there'-no one could really perform some alternative measurement on the other wing of the experiment without changing the entire spatiotemporal description of the experiment. 
Thus, correlations between space-like separated events that violate Bell's inequalities are of no concern as long as spacetime symmetries instantiated by the experimental apparatus warrant the correlated spacetime relations. Since the non-local correlations derive from the spatiotemporal relations per the spacetime symmetries of the experiment, satisfaction of any common-cause principle is superfluous.

Our account provides a clear description, in terms of fundamental spacetime relations in a blockworld, of quantum phenomena that does not suggest the need for a "deeper" causal or dynamical explanation. If explanation is simply determination, then our view explains the structure of quantum correlations by invoking what can be called acausal, global determination relations. These global determination relations are given by the spacetime symmetries which underlie a particular experimental setup. Not objects governed by dynamical laws, but rather acausal spacetime relations per the relevant spacetime symmetries do the fundamental explanatory work according to our version of geometrical quantum mechanics. We can invoke the entire spacetime configuration of the experiment so as to predict, and explain, the EPR-Bell correlations. Indeed, it has been the purport of this paper that the spacetime symmetries of the quantum experiment can be used to construct its quantum density operator, that such a spacetime is one for which simultaneity is relative, and that events in the detector regions evidence spatiotemporal relations.

\section{REFERENCES}

1. Murray Gell-Mann in L. Wolpert, The Unnatural Nature of Science, Cambridge: Harvard University Press, 1993, p. 144.

2. G. Kaiser, J. Math. Phys. 22, 705-714 (1981); G. Kaiser, Quantum Mechanics, Relativity, and Complex Spacetime: Towards a New Synthesis, Amsterdam: North-Holland Press, 1990.

2. A. Bohr and O. Ulfbeck, Rev. Mod. Phys. 67, 1-35 (1995).

3. J. Anandan, Int. J. Theor. Phys. 42, 1943-1955 (2003).

5. A. Bohr, B. Mottelson and O. Ulfbeck, Found. Phys. 34, 405-417 (2004); A. Bohr, B. Mottelson and O. Ulfbeck, Phys. Today 57, 15-16 (2004).

6. R. Carnap in P. Schilpp (ed.), The Philosophy of Rudolf Carnap, La Salle, IL: Open Court, 1963, pp. 3-84.

7. R. Feynman, R. Leighton and M. Sands, The Feynman Lectures on Physics, Vol. III, Quantum Mechanics, Reading: Addison-Wesley, 1965, p. 1-1.

8. H. Brown and P. Holland, Am. J. Phys. 67, 204-214 (1999).

9. A derivation of Eq. (1), assuming the acceptability of a phase difference such as that in Eq. (4), is in L. Ballentine, Quantum Mechanics, Englewood Cliffs: Prentice Hall, 1990, pp. 49-58.

10.H. Georgi, Lie Algebras in Particle Physics, $2^{\text {nd }}$ Ed., New York: Perseus Books, 1999, p. 18.

11. A. Zee, Quantum Field Theory in a Nutshell, Princeton: Princeton University Press, 2003, p. 18. 